\title{
FREQUÊNCIA FUNDAMENTAL DE CRIANÇAS DA CIDADE DE NITERÓI
}

\section{Fundamental frequency for children in the Municipality of Niterói}

\author{
Tereza Cristina Andrade Schott ${ }^{(1)}$, Tania Maria Marinho Sampaio (2), \\ Domingos Sávio Ferreira de Oliveira ${ }^{(3)}$
}

\section{RESUMO}

Objetivo: medir a frequência fundamental de 122 crianças de seis a oito anos de idade, meninos e meninas, sem alterações vocais, avaliadas por meio da escala Rasat. Métodos: a análise perceptivo-auditiva foi realizada através da escala RASAT e para a análise acústica computadorizada foi utilizado o programa de software Vox Metria, analisando-se a média da frequência fundamental de amostras da vogal [ $[$ ] sustentada. Participaram deste estudo 122 crianças, 58 meninos e 64 meninas com idade variando de seis a oito anos e 11 meses de idade do ensino fundamental, da Rede Municipal de Niterói-RJ, nascidos e residentes na cidade, pré-selecionadas através de um protocolo de triagem. Resultados: os resultados obtidos neste estudo foram: frequência fundamental média de $239,60 \mathrm{~Hz}$ para as meninas e $237,17 \mathrm{~Hz}$ para os meninos, obtendo-se uma média geral de 238,44 $\mathrm{Hz}$. Conclusão: conclui-se que os achados permitiram a comparação com resultados de pesquisas realizadas anteriormente, contribuindo com novos dados para a padronização da frequência fundamental das vozes infantis brasileiras.

DESCRITORES: Medidas; Criança; Voz

\section{INTRODUÇÂO}

A frequência fundamental é determinada pela tensão das pregas vocais pelos músculos da laringe que são controlados pelo nervo vago (X par craniano), tendo a variabilidade da frequência fundamental controlada pela coordenação laríngea ${ }^{1}$. Esta medida é o resultado da interação entre o comprimento, a massa e a tensão das pregas vocais durante a fonação. Acredita-se que entre cinco e dez anos seja o período onde se tem o maior índice de disfonia infantil ${ }^{2}$. Crianças em idade escolar

(1) Fonoaudióloga; Clínica Tereza Schott; Mestre em Fonoaudiologia pela Universidade Veiga de Almeida.

(2) Graduação em Letras pela Universidade Federal do Rio de Janeiro; Professora da Universidade Veiga de Almeida, UVA, Rio de Janeiro, RJ; Professora da Universidade Federal Fluminense, UFF, Niterói, RJ; Doutora em Filosofia pela Universidade Gama Filho.

(3) Fonoaudiólogo; Diretor-Pesidente da Clínica da Voz; Professor da Universidade Veiga de Almeida, UVA, Rio de Janeiro, RJ; Professor da Universidade do Estado do Rio de Janeiro, UERJ, Rio de Janeiro, RJ; Doutor em Linguística pela Universidade Fluminense Federal.

Conflito de interesse: INEXISTENTE apresentam problemas de hiperfuncionamento vocal com mais frequência ${ }^{3}$. Constitui-se este um desafio necessário ao fonoaudiólogo analisar a voz da criança.

O entendimento da fisiologia e da acústica fonatória é a base para descrever as características de uma voz, o que necessita de vários procedimentos, como as avaliações perceptivo-auditiva e acústica. As análises baseiam-se na percepção auditiva dos avaliadores, contando com padronizações em escalas ${ }^{4}$. Nesta pesquisa utiliza-se a RASAT ${ }^{5}$, uma versão brasileira adaptada da escala GRBAS ${ }^{6}$.

Dentre os vários parâmetros vocais acústicos que podem ser obtidos através de programas ou equipamentos específicos para esta função, a frequência fundamental (f0) se destaca como um dos parâmetros mais importantes. Corresponde ao número de ciclos de vibração por segundo das pregas vocais e sua extração tem se mostrado o parâmetro mais resistente aos diversos sistemas de análise acústica ${ }^{7}$. No campo da exploração das medidas acústicas, a literatura aponta a necessidade de padronizações de procedimentos e valores normativos ${ }^{8}$. 
A análise da frequência fundamental é de grande importância para o estudo do desenvolvimento da voz em função da idade, e já foi estudada por diversos autores ${ }^{9,10}$ que buscaram uma normatização desta medida em crianças, mulheres e homens. É nesta direção que se dá uma abertura à pesquisa: verificar se a frequência fundamental da criança brasileira é semelhante à média de frequência encontrada na literatura internacional.

As pregas vocais são mais flexíveis na superfície e tornam-se mais rígidas em direção ao músculo vocal, característica essencial para o adequado movimento vibratório das pregas vocais ${ }^{11}$.

É importante considerar que, do nascimento até a puberdade, ocorrem significativas mudanças na estrutura laríngea. As pregas vocais aumentam de tamanho e massa, modificando as proporções cartilaginosas e membranosas das mesmas. Primariamente, a frequência fundamental relaciona-se com o comprimento das pregas vocais, o que leva a concluir que a frequência fundamental diminui na medida em que a criança cresce, considerando não apenas o aumento da laringe, mas também a maturação do sistema nervoso, o que possibilita maior controle laríngeo ${ }^{12}$.

As medidas de frequência fundamental pesquisadas em outros países evidenciam uma faixa de frequência diferente das obtidas nas pesquisas nacionais: Nessas, uma criança de oito anos de idade possui uma frequência fundamental de aproximadamente $275 \mathrm{~Hz}{ }^{13}$. Quanto ao português brasileiro falado na cidade de São Paulo, constataram-se valores de $235,8 \mathrm{~Hz}$ para crianças ${ }^{14}$. Na pesquisa realizada em Porto Alegre ${ }^{15}$, a média encontrada para ambos os sexos é de $250,05 \mathrm{~Hz}$. No Brasil, os valores de referência utilizados para crianças é de $250 \mathrm{~Hz}$ para as crianças ${ }^{8}$.

Uma das características de problemas vocais nas crianças é o nível de frequência fundamental ou muito alto ou muito baixo relacionado à idade $e$ ao sexo ${ }^{16}$.

A frequência hiperaguda também pode ser encontrada em doenças congênitas e do sistema nervoso ${ }^{17}$.

A normatização das medidas acústicas é necessária para conhecer melhor a voz normal ${ }^{18}$. Dessa forma, o objetivo dessa pesquisa é quantificar a frequência fundamental de crianças de seis a oito anos de idade, meninos e meninas, sem alterações vocais significativas, ou seja, crianças com um discreto grau de alteração da qualidade vocal, de normal para leve, na avaliação perceptivo-auditiva, através da escala RASAT ${ }^{5}$, não foram afastadas da pesquisa.

\section{MÉTODOS}

Esta pesquisa tem caráter quali-quantitativa, objetivando analisar a frequência fundamental em relação à idade e ao sexo dos participantes, alunos da Rede Municipal da Cidade de Niterói, do Estado do Rio de Janeiro. Participaram 122 crianças, 58 meninos e 64 meninas com idade variando de seis a oito anos e 11 meses de idade do ensino fundamental, da Rede Municipal de Niterói-RJ, nascidos e residentes na cidade, pré-selecionadas através de um protocolo de triagem foram excluídas crianças com distúrbios vocais ou história de disfonia e doenças correlacionadas, investigadas através do referido protocolo e análise percepto-auditiva (RASAT) ${ }^{5}$.

Foram utilizados os seguintes instrumentos:

- Formulário adaptado da Avaliação da Clínica de Otorrinolaringologia e Cirurgia de Cabeça e Pescoço (Stefen, 1997)

- Análise perceptivo-auditiva (RASAT) ${ }^{5}$

- Análise acústica computadorizada (Voxmetria 2.6)

- Notebook HP Pavillion ZE4120, com um processador AMD Semprom Móbile 3000, 1.8Ghz, memória de sistema de $256 \mathrm{MB}$, disco rígido de 40GB, Microsoft Windows XP Home e placa de som Sound Blaster.

- Microfone da marca Shure Sm 58 (unidirecional).

- Tripé de mesa.

- Medidor de nível sonoro (Realistic-Tandy) Foram seguidos os seguintes procedimentos: Gravação das vozes das crianças, contando de um a dez para análise perceptivo-auditiva. Todas as vozes passaram por uma análise perceptivoauditiva com três fonoaudiólogas juradas, especialistas em voz ${ }^{19}$. Para análise acústica, cada criança foi orientada a respirar antes de realizar a tarefa de emitir a vogal $[\varepsilon]$ de forma isolada e sustentada em nível de frequência, intensidade e qualidade vocais confortáveis. De cada criança foi considerado, como amostra vocal, um trecho médio de dois a três segundos, o mais estável possível, de três repetições. $\mathrm{Na}$ análise da amostra foi excluído o início e o término para que o ataque e/ou decréscimo da intensidade não interferissem na análise. A criança estava sentada e o microfone fixado ao tripé, posicionado ao redor de três a quatro centímetros de distância da altura da sua boca, durante a sustentação da vogal $[\varepsilon]$.

Para ter certeza de que as crianças entenderam a tarefa foi proposto um exemplo inicial.

Cada criança, durante a gravação, estava de costas para a tela do computador, para que não ficasse dispersa com a imagem do monitor. Utili- 
zou-se um elástico nas mãos para sustentar mais tempo de fonação, esticando-o enquanto emitia a vogal.

A escolha da vogal $[\varepsilon]^{20}$ é critério utilizado pelo programa, por ser a vogal mais fielmente expressa pelo número de semitons da emissão. Esta tem sido considerada a vogal mais estável do ponto de vista laríngeo nas vozes do português brasileiro ?

Para recortar as amostras, verifica-se a espectrografia e a intensidade da amostra vocal. Utiliza-se o diagrama de desvio fonatório, que é um método para descrever a periodicidade dos ciclos glóticos e o conteúdo de ruído das vozes, com a finalidade de expressar graficamente a análise da qualidade vocal. A medida da f0 foi extraída através dos dados da análise vocal, onde é expresso o resultado estatístico da média da f0.

Este trabalho foi aprovado pelo Comitê de Ética em Pesquisa da Universidade Veiga de Almeida, sob o número resolução 115/08.

A análise estatística aplicada sustenta-se no uso do "t" student que foi utilizado para comparar as médias das idades e sexos, adotando-se nível de significância de 0,05.

\section{RESULTADOS}

Os resultados foram tratados estatisticamente, visando a obtenção da média da frequência fundamental (f0) na população avaliada, por idade e sexo, como também o desvio-padrão (d.p) e a comparação inter e intra grupos dos valores obtidos.

$\mathrm{Na}$ Tabela 1 as médias da f0 média, obtidas foram $239,60 \mathrm{~Hz}$ para as meninas e $237,17 \mathrm{~Hz}$ para os meninos, tendo como média geral de ambos os sexos $238,44 \mathrm{~Hz}$.

Levantando os dados por idade e sexo, Tabela 2, obtiveram-se os seguintes resultados: meninos de seis anos, $241,41 \mathrm{~Hz}$; meninas de seis anos, 251,08 Hz; meninos de sete anos, $233,26 \mathrm{~Hz}$; meninas de sete anos, $234,08 \mathrm{~Hz}$; meninos de oito anos, 239,40 Hz; meninas de 8 anos, $233,19 \mathrm{~Hz}$.

\section{DISCUSSÃO}

Das 122 crianças avaliadas, 13 apresentaram um discreto grau de alteração da qualidade vocal, de normal para leve, na avaliação perceptivo-auditiva, através da escala RASAT, mas não foram afastadas da pesquisa, pois um discreto grau de rouquidão, nasalidade e/ou soprosidade pode ser considerado como a "voz esperada" na infância 7 . Os diagramas de desvio fonatório destas 13 crianças apresentaram-se discretamente fora da área de normalidade, confirmando a avaliação perceptivo-auditiva.

As amostras vocais colhidas para análise acústica neste estudo correspondem a sinais tipo 1 que, como citado ${ }^{7}$, são quase periódicos, sem alterações qualitativas no segmento analisado. Vozes normais ou com pequenas alterações, geralmente produzem sinal deste tipo.

O comprimento das pregas vocais ${ }^{13}$ é de 6-8 $\mathrm{mm}$ nas crianças. Segundo autores ${ }^{7}$ vai de 4,5 a $5,5 \mathrm{~mm}$ nas meninas e de 5,7 a $7 \mathrm{~mm}$ nos meninos. Portanto, observam-se diferenças, inclusive em relação ao sexo, uma vez que as medidas das meninas e meninos brasileiros apresentam-se abaixo das medidas das crianças americanas.

Tal diferença, em relação ao tamanho das pregas vocais, leva a pensar nos estudos populacionais, onde se destacam alguns referenciais construídos em outros países, que são utilizados como parâmetros de análise em todas as regiões. Dentre estes, a Organização Mundial de Saúde recomenda as curvas do National Center For Health Statistics (NCHS), elaboradas com base no crescimento de crianças norte americanas. No Brasil, dois estudos tentaram construir um padrão nacional de crescimento. Um estudo ${ }^{21}$, realizado com a população de Santo André e da Grande São Paulo, constatou que um subgrupo da amostra, composto por crianças pertencentes a famílias de renda alta, era similar aos resultados encontrados pelo "National Center For Health Statistics".

A quinta conferência Internacional sobre Promoção da Saúde, realizada no México, em 2000 (MINISTÉRIO DA SAÚDE 2002), tratou de temas como a necessidade de possibilitar maior impacto na saúde mundial, por meio da formação de uma aliança entre países mais populosos, levando-se

Tabela 1 - Média da frequência fundamental geral

\begin{tabular}{c|ccc}
\hline Sexo & Média f0 $(\bar{X})$ & significância & № de crianças \\
\hline Feminino & 239,60 & 0,05 & 64 \\
Masculino & 237,17 & 0,05 & 58 \\
\hline
\end{tabular}

Conclui-se haver diferença significativa entre as frequências fundamentais de ambos os sexos ao nível de significância de $5 \%$. 
Tabela 2 - Média da frequência fundamental por sexo e idade

\begin{tabular}{|c|c|c|c|}
\hline \multirow{3}{*}{ Idade (anos) } & \multicolumn{3}{|c|}{ Média da f0 (Hz) } \\
\hline & \multicolumn{2}{|c|}{ Sexo } & \multirow{2}{*}{ Média Geral } \\
\hline & Feminino & Masculino & \\
\hline 6 & 251,08 & 241,41 & 247,16 \\
\hline 7 & 234,08 & 233,26 & 233,61 \\
\hline 8 & 233,19 & 239,40 & 235,83 \\
\hline Média Geral & 239,60 & 237,17 & 238,44 \\
\hline
\end{tabular}

a compreender o conceito da padronização das medidas da voz, dentro de um contexto mais amplo. Alguns autores assinalaram que informações vocais que dizem respeito a dados físicos, como sexo, idade e condições gerais de saúde, estariam contidas na dimensão biológica ${ }^{22}$.

Nos países em desenvolvimento, como o Brasil, existem diferenças no padrão de vida entre as regiões e, até mesmo, dentro da mesma região. Assim, torna-se relevante a realização de pesquisas em diferentes populações e regiões do Brasil ${ }^{21}$.

As médias da fo média, Tabela 1, obtidas na presente pesquisa, foram $239,60 \mathrm{~Hz}$ para as meninas e $237,17 \mathrm{~Hz}$ para os meninos, tendo como média geral de ambos os sexos $238,44 \mathrm{~Hz}$. Estas medidas se aproximam da pesquisa realizada em São Paulo ${ }^{2}$ que obteve uma média de $235,8 \mathrm{~Hz}$. $\mathrm{Na}$ pesquisa realizada em Porto Alegre ${ }^{15}$ a média encontrada para ambos os sexos foi de $250,05 \mathrm{~Hz}$, percebendo-se aí uma diferença.

As médias encontradas na literatura internacional apresentam uma diferença significativa, de $297 \mathrm{~Hz}$ para meninos e $286,5 \mathrm{~Hz}$ para as meninas ${ }^{23}$. Uma criança de oito anos possui uma f0 em aproximadamente $275 \mathrm{~Hz}^{13}$.

No presente estudo, levantando os dados por idade e sexo, Tabelas 3 e 4 , foram obtidos os seguintes resultados: meninos de seis anos, $241,41 \mathrm{~Hz}$; meninas de seis anos, $251,08 \mathrm{~Hz}$; meninos de sete anos, $233,26 \mathrm{~Hz}$; meninas de sete anos, $234,08 \mathrm{~Hz}$; meninos de oito anos, $239,40 \mathrm{~Hz}$; meninas de oito anos, $233,19 \mathrm{~Hz}$. Pode-se observar uma diferença maior na idade de sete anos, em ambos os sexos. A relevância de tal diferença torna-se um significativo dado a ser estudado em pesquisas futuras.

Existem outros estudos que citam tais diferenças ${ }^{4}$, onde afirmam os autores que vários estudos relacionam as medidas de CV (capacidade vital respiratória) de crianças, com as diferentes faixas de idade, estatura, tamanho de caixa torácica dos sujeitos e peso ${ }^{24,25}$.

Segundo o Consenso sobre Espirometria, a estatura é o fator que exerce a maior influência sobre a CV na infância ${ }^{26}$.

Foram relatadas diferenças em algumas medidas de dinâmica respiratória entre crianças de escolas particulares e públicas. Sabe-se que, atualmente, no Brasil, pode-se encontrar grande diferença na situação socioeconômica e cultural entre os alunos de escolas públicas e particulares ${ }^{26}$.

A população aqui avaliada é de escola pública, de classe social de baixa renda; já na de Porto Alegre, segundo a pesquisa do INAN, as crianças pertenciam a famílias de renda alta e apresentavam estatura avaliada por uma fita métrica, fixada à parede, com o ponto zero no nível do solo ${ }^{21}$. Observou-se estatura semelhante a das crianças norte americanas.

Fica ainda, como outra frente desta pesquisa, a possível constatação (ou não), da hipótese de que, crianças de baixa renda, por terem, devido a fatores nutricionais e genéticos, estatura menor, apresentariam também cartilagens menores e menor comprimento de pregas vocais, bem como ressonadores naturais do corpo de menor tamanho, deixando ainda em aberto, neste estudo, questões que nele próprio se atravessaram.

\section{CONCLUSÃO}

As médias da f0 média de crianças de 6 a 8 anos, sem alterações vocais, obtidas na presente pesquisa, foram $239,60 \mathrm{~Hz}$ para as meninas e $237,17 \mathrm{~Hz}$ para os meninos, tendo como média geral de ambos os sexos $238,44 \mathrm{~Hz}$, evidenciando valores abaixo das médias encontradas na literatura internacional. 


\begin{abstract}
Purpose: to quantify the fundamental frequency in a group of 122 children - boys and girls, whose age varied from six to eight-year-old, who do not have vocal alterations. Methods: an auditory-perceptive analysis took place by means of the RASAT scale; the computerized acoustic analysis was performed by using a software referred to as Vox Metria, where samples regarding the mean value for the fundamental frequency of the sustained vowel [0] were analyzed. For this study, in which 122 children took part, 58 boys and 64 girls, with ages ranging from 6 to 8 -year and 11 months. They were students from the Elementary Schools of the Municipal Education System of Niteroi-RJ. They were born and reside in this municipality and were pre-selected by means of a choice protocol. Results: the results obtained in this study were $239.60 \mathrm{~Hz}$ for the girls and $237.17 \mathrm{~Hz}$ for the boys, with an overall mean value of $238.44 \mathrm{~Hz}$. Due to the small difference; we obtained $237.57 \mathrm{~Hz}$ for the girls and $233.31 \mathrm{~Hz}$ for the boys. Conclusion: the findings enabled the comparison with previously carried out research and contributed providing the literature with new data for the standardization of the fundamental frequency of Brazilian Children's Voices, opening a new channel for further research.
\end{abstract}

KEYWORDS: Measure; Child; Voice

\section{REFERÊNCIAS}

1. Branco A, Fekete SMW, Rugolo LMSS, Rehder MI. Valor e variações da frequência fundamental no choro de dor de recém-nascidos. Rev CEFAC. 2006; 8(4):529-35.

2. Teixeira MZM, Trezza EMC, Behlau M. Opinião dos pais sobre a voz de seus filhos de 5 a 12 anos. Rev Paul Pediatr. 2003; 21(2):68-75.

3. Spinelli ICP. Medidas fonatórias de crianças de uma escola pública do Município de São Paulo [dissertação] São Paulo (SP): Universidade de São Paulo; 2004.

4. Sader RCM, Hanayama EM. Considerações teóricas sobre a abordagem acústica da voz infantil. Rev CEFAC. 2004; 6(3):312-8.

5. Pinho SMR, Pontes P, Pinho SM, Pontes P. Escala de avaliação perceptiva da fonte glótica: RASAT. Vox Brasilis. 2002; 8(3):11-3.

6. Hirano M. Clinical examination of voice. New York: Springer-Verlag; 1981.

7. Behlau MS, Feijó D, Madazio G, Brasil O, Pontes P, Azevedo R. Avaliação de voz. In: Behlau MS, organizador. Voz: o livro do especialista. São Paulo: Revinter; 2001. p.85-180.

8. Sader RCM, Hanayama EM. Análise acústica da voz infantil. Rev CEFAC. 2004; 6(3):312-8.

9. Fairbanks G. An acoustical study of the pitch of infant hunger wails. Child Dev. 1942; 13:227-32.

10. Wilson K. Problemas da voz em crianças. 3. ed. São Paulo: Manole; 1994.

11. Braga NJ, Domingos SFO, Atherino CCT, Schott TCA, Silva JS. Nódulos vocais: análise anátomofuncional. Rev CEFAC. 2006; 8(2):223-9.
12. Titze I, Horii Y, Scherer R. Some technical considerations in voice pertubation measurements. Resp Rep DCPA-RR1. Denver: The Denver Center of Performing Arts; 1985. p.50-78.

13. Sataloff R. Professional voice: the science and art of clinical care. 2. ed. San Diego-London: Singular Publishing Group; 1997.

14. Behlau M, Tosi $O$, Pontes P. Determinação da frequência fundamental e suas variações em altura ("jitter") e intensidade ("shimmer") para falantes do português brasileiro. Acta AWHO. 1985; 4(1):5-10.

15. Steffen N, Moschetti M. Parâmetros acústicos de Jitter e Shimmer de 248 crianças de 6 a 10 anos, estudantes de Porto Alegre. Rev Bras Otorrinolaringol. 1997; 63(4):329-34.

16. Wilson KD. Voice problems of children. 3. ed. Baltimore: The Williams e Wilkins Co; 1987. p.4-6.

17. Branco A, Fekete SMW, Rugolo LMSS. Valor e variação da frequência fundamental no choro de dor de recém nascidos. Rev CEFAC. 2006; 8(4):529-35.

18. Araújo SAA, Grellet M, Pereira JC, Rosa MO. Normatização das medidas acústicas da voz normal. Rev Bras Otorrinolaringol. 2002; 68(4):540-4.

19. Beneton G, Botelho SW, Silva L, Castro PC. Comparação dos parâmetros vocais em crianças com e sem rinite alérgica. Rev Fono Atual. 2004; 7(29):30-9.

20. Andrade LMO. Determinação dos limiares de normalidade dos parâmetros da voz. [dissertação] São Paulo (SP): Universidade de São Paulo; 2003. 21. Diniz IMS, Lopes AS, Dummel CCB, Rieger T. Crescimento físico e adiposidade corporal de escolares. Rev Bras Cineantrop Desemp Hum. 2006; 8(2):32-8. 
22. Spinelli ICP. Medidas fonatórias de crianças de uma escola pública do município de São Paulo. [dissertação]. São Paulo (SP): Universidade de São Paulo; 2004.

23. Fairbanks G, Wiley JH, Lassman FL. An acoustical study of vocal pitch in seven- and eightyear-old boys. Child Dev. 1949; 20(2):63-9.

24. Zemlin WR. Princípios de anatomia e fisiologia em fonoaudiologia. Porto Alegre: Artes Médicas Sul; 2000.

25. Subbarao P, Lebecque P, Corey M, Coates AL. Comparison of spirometric reference values. Pediatr Pulmonol. 2004; 37(6):515-22.

26. Fabron EMG, Santos GR, Omote S, Perdoná GC. Medidas da dinâmica respiratória em crianças de quatro a dez anos. Pró-Fono. 2006; 18(3):313-22.

RECEBIDO EM: 05/05/2008

ACEITO EM: 24/11/2008

Endereço para correspondência:

Tereza Cristina Andrade Schott

Rua Coronel Moreira César, 26 sala 911

Niterói - RJ

CEP: 24230-060

E-mail: teteschott@gmail.com 